\title{
Deep, wide-field global VLBI observations of the HDF-N
}

\section{Seungyoup Chi*}

Kapteyn Institute, Rijksuniversiteit Groningen, P.O.Box 800, 9700 AV, Groningen, The Netherlands Joint Institute for VLBI in Europe (JIVE), Postbus 2, 7990 AA, Dwingeloo, The Netherlands

E-mail: chicastro.rug.nl

\section{Michael A. Garrett}

Joint Institute for VLBI in Europe (JIVE), Postbus 2, 7990 AA, Dwingeloo, The Netherlands

\section{Peter D. Barthel}

Kapteyn Institute, Rijksuniversiteit Groningen, P.O.Box 800, 9700 AV, Groningen, The Netherlands

\begin{abstract}
We present initial results of wide-field Global VLBI 1.4-GHz observations of the Hubble Deep Field North region (HDF-N) and Flanking Fields surrounding it with a spatial resolution of 4 milliarcseconds. These observations are both deeper and wider than any previous VLBI observations of the field, attaining an r.m.s. noise level of $7.3 \mu \mathrm{Jy} /$ beam in the central field. Four radio sources have been clearly detected in the inner part of the field and a search for additional sources in the adjacent Hubble Flanking Field (HFF) is underway. Among the four detections in the HDF-N, three (VLA J123642+621331, VLA J123644+621133, and VLA J123646+621404) have already been detected in previous EVN 1.6-GHz observations, but the forth one, VLA $\mathrm{J} 123652+621444$, is a new detection that clearly shows one-sided, jet-like radio structure. The VLBI observations of VLA J123642+621331 - classified at other wavelengths as a distant, dust-obscured starburst galaxy at $z=4.424$ - reveal a resolved jet-like extension emanating from a compact AGN core. These results demonstrate the power of high-resolution VLBI imaging in discriminating between starburst and AGN activity in dust-obscured systems. We plan to analyse 80 additional radio target fields in the HFF over the next few months ${ }^{1}$.
\end{abstract}

8th European VLBI Network Symposium

September 26-29, 2006

Torun, Poland

\footnotetext{
* Speaker.

${ }^{1}$ At the time of writing this proceeding, we have finished the data reduction and 8 more objects have been detected in the HFF.
} 


\section{Introduction}

Deep, high-sensitivity radio observations of the Hubble Deep Field North (HDF-N) and surrounding Flanking Fields (HFF) have revealed a population of faint sub-mJy and $\mu J y$ radio sources for the majority of which are associated with relatively distant, star-forming galaxies (Richards et al. 1998, Garrett et al. 2001, Muxlow et al. 2005). Radio-to-optical comparison studies of this field demonstrate that a significant fraction of these sources are optically-faint, remaining undetected in deep HST I-band imaging. The optically-faint systems are thought to be distant galaxies obscured by dust. Deep, high-resolution VLBI observations can throw light on the nature of these systems, discriminating (in terms of component brightness temperature) whether radio emission properties are dominated by star formation or AGN processes. Such studies are also useful in establishing the relationship between star formation and AGN activity in high-redshift systems. In this contribution, we present the first results of deep, wide-field global VLBI 1.4-GHz observations of the HDF-N (and HFF). These observations have an angular resolution of 4 milliarcseconds and an r.m.s. noise level of $7.3 \mu \mathrm{Jy} / \mathrm{beam}$ in the field centre.

\section{Global VLBI observations and data analysis}

Observations of the HDF-N and HFF regions were made with global VLBI network on 20-22 February 2004 at $1.4 \mathrm{GHz}$. A total of 36 hours observing time was split into three, 12hour runs. The global VLBI network consisted of 16 telescopes in Europe and the USA, including the 100-m Effelsberg (DE), 100-m Green Bank (US), and 76-m Lovell (UK) telescopes; recording data at a bit rate of $64 \mathrm{Mbits} / \mathrm{s}(2 \times 8-\mathrm{MHz}$ bands $)$ in both right (R)- and left (L)-hand circular polarisation. The observations were made in phase-reference mode using two phase-calibrators: the primary calibrator, J1241+602 and a fainter, secondary calibrator, $\mathrm{J} 1234+619$.

The data were processed using the EVN correlator at JIVE. Each 8-MHz band (R\&L) was correlated in two separate passes in order to maximise spectral resolution and so to minimise bandwidth smearing. An integration time of 0.25 seconds was adopted to reduce the effects of time smearing. The phase centre was coincident with a $470-\mu \mathrm{Jy}$ radio source, VLA J123642+621331 (Richards 2000), lying just outside the HDF-N. All subsequent data processing was carried out with the NRAO AIPS package.

In order to image out the entire HDF-N and HFF, the wide-field imaging technique was used (Garrett et al. 1999). Dirty images from each 8-MHz band (R\&L simultaneously) were generated; fields with a clear detection were then CLEANed using APCLN. Images from each 8-MHz band (and each 12-hour observing run) were then co-added. Images in the HDF-N attain an r.m.s. noise level of $7.3 \mu \mathrm{Jy} / \mathrm{beam}$, consistent with the predicted thermal noise level. 


\section{Global VLBI detections}

The global VLBI observations clearly revealed four radio sources in the inner part of the field above the $10 \sigma$ detection level (Figure 1). Among the four detections in the HDF-N, three (VLA J123642+621331, VLA J123644+621133, and VLA J123646+621404) were already detected in earlier EVN 1.6-GHz observations (Garrett et al. 2002), but the forth one, VLA $\mathrm{J} 123652+621444$, is a new detection that clearly shows one-sided, jet-like radio structure.

\subsection{VLA J123642+621331}

VLA J123642+621331 lies just outside the HDF-N, in an adjacent HFF. It has no optical counterpart down to $I=25$ mag. Waddington et al. (1999) detected a very red counterpart to the radio source with NICMOS in $J$ and $H$ bands, and with the KPNO 4-m in $K$ band. Based on a single Ly $\alpha$ emission line, they obtained a redshift of 4.424 and interpreted this source as a dustobscured starburst with an embedded AGN.

The MERLIN-VLA observations at $1.4 \mathrm{GHz}$ (Muxlow et al. 2005) resolved VLA $\mathrm{J} 123642+621331$, showing extended emission adjacent to an unresolved core. It was the core which was detected by the EVN at $1.6 \mathrm{GHz}$ (Garrett et al. 2001). The ratio of its radio and FIR luminosity indicates that the object is radio-loud (Garrett 2002), implying the existence of an embedded AGN. The new global VLBI images confirm this, revealing a resolved jet-like extension emanating from a compact AGN core. This may be an example of a high-redshift ULIRG in which the high star-formation rate and efficiency is supported by AGN jet activity (e.g. Silk 2005).

\subsection{VLA J123644+621133}

VLA J123644+621133 is identified with a bright, red elliptical galaxy at $z=1.013$. Its large-scale radio structure and luminosity exhibit a classical FR-I structure (Richards et al. 1998; Muxlow et al. 2005). The previous EVN observations (Garrett et al. 2001) revealed a compact core component and an additional $5 \sigma$ component located $\sim 60$ mas south of the core component. They suggested that this southern faint component was a knot in the extended MERLIN-VLA jet. However, in our new global VLBI observations, we only detect the compact core. We plan to produce tapered images of this source in case the higher-resolution global observations resolve out any extended structure associated with this source.

\subsection{VLA J123646+621404}

VLA J123646+621404, located within the HDF-N itself, is associated with a very red, face-on spiral galaxy at $z=0.960$, and has a flat radio spectrum $(\alpha=-0.04)$. Rowan-Robinson et al. (1997) interpreted this source as a massive starburst, based on the ISO 7- $\mu \mathrm{m}$ emission. But the other properties of VLA J123646+621404 (the presence of MgII emission, radio variability, and strong X-ray emission) suggest the spiral also hosts an AGN. The compact core component detected in both the original EVN and the new global VLBI observations supports this hypothesis. 


\subsection{VLA J123652+621444}

VLA J123652+621444 is located just outside of the HDF-N. It has an inverted spectrum $(\alpha=-0.12)$ and is one of the few radio sources in the field observed to vary in intensity on short (monthly) time-scales (Richards et al. 1998). The MERLIN-VLA images show a compact core and one-sided, jet-like feature to the east. The source was not revealed in the original EVN observations (above the $3 \sigma$ level) but the much deeper global VLBI observations clearly detect a $70 \mu \mathrm{Jy}$ radio source.

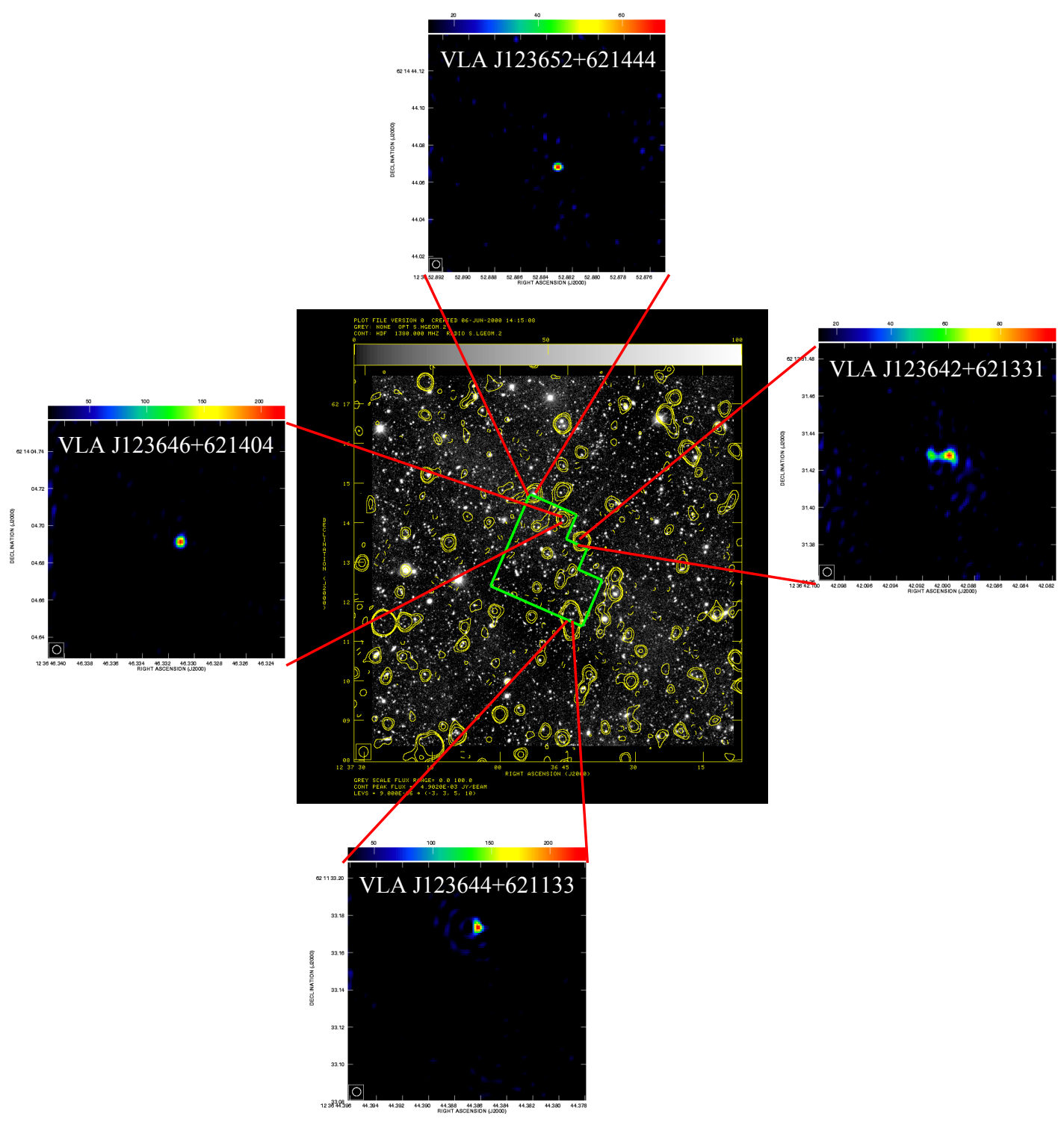

Figure 1. The global VLBI 1.4-GHz images of four detections located in the inner part of the HDF-N and HFF. The centre image is WRST 1.4-GHz image with CFHT I-band image (Garrett et al. 2000). 


\section{Conclusions}

New deep, wide-field global VLBI observations have revealed four radio sources in the inner part of the HDF-N. The images attain an r.m.s. noise level of $7.3 \mu \mathrm{Jy} / \mathrm{beam}$. Among the four detections in the field, three objects (VLA J123642+621331, VLA J123644+621133, and VLA J123646+621404) have been detected in previous EVN 1.6-GHz observations. VLA $\mathrm{J} 123652+\mathrm{J} 621444$ (known to be variable from VLA observations) is a new detection. The highresolution images of VLA J123642+621331 - classified at other wavelengths as a distant, dustobscured starburst galaxy at $z=4.424$ - reveal a resolved jet-like extension emanating from a compact AGN core. The role this jet plays in initiating or regulating star formation in this system is unclear. At the time of the this meeting, 80 additional radio targets in the HFF are ready to be analysed. Based on previous wide-field VLBI surveys, we expect at least half-adozen additional sources to be detected in the HFF. The results presented here clearly demonstrate the power of deep, high-resolution VLBI imaging in discriminating between starburst and AGN activity in dust-obscured systems.

\section{References}

[1] Garrett, M. A., Porcas, R. W., Pedlar, A., Muxlow, T. W. B., and Garrington, S. T., 1999, NewAR, 43, 519 [astro-ph/9906108]

[2] Garrett, M. A., de Bruyn, A. G., Giroletti, A. G., et al., 2000, A\&A, 361, L41

[3] Garrett, M. A., Muxlow, T. W. B., Garrington, S. T., et al., 2001, A\&A, 366, L5

[4] Garrett, M. A., 2002, A\&A, 384, L19

[5] Muxlow, T. W. B., Richards, A. M. S., Garrington, S. T., et al., 2005, MNRAS, 358, 1159

[6] Richards, E. A., Kellermann, K. I., Fomalont, E. B., et al. 1998, AJ, 116, 1039

[7] Richards, E. A., 2000, ApJ, 533, 611

[8] Silk, J., 2005, MNRAS, 364, 1337 